\title{
Field test results of compound specific isotope analyzer based on quantum cascade lasers and hollow waveguide
}

Sheng Wu, Andrei Deev

Sheng Wu, Andrei Deev, "Field test results of compound specific isotope analyzer based on quantum cascade lasers and hollow waveguide," Proc. SPIE 9370, Quantum Sensing and Nanophotonic Devices XII, 937010 (8 February 2015); doi: 10.1117/12.2080737

SPIE. Event: SPIE OPTO, 2015, San Francisco, California, United States 


\title{
Field test results of compound specific isotope analyzer based on quantum cascade lasers and hollow waveguide
}

\author{
Sheng $\mathrm{Wu}$, Andrei Deev \\ PEER Institute \\ 738 Arrow Grand Circle, Covina, CA 91722
}

\begin{abstract}
The first of its kind Gas Chromatograph Infra Red Isotope Ratio (GC-IR2) instruments have been deployed to the field to help the identification of sweet spot during the shale gas exploration. The onsite measurement capability of the GC-IR2 along with its accuracy and speed helped the discovery of the fast dynamics of gas release from shale cuttings. The half life of the isotope change for methane, ethane and propane released from shale cuttings is closely related to the porosity and permeability of the specific shale reservoir, and could be as short as a one hour to a couple of days. Initial $\delta^{13} \mathrm{C}$ values for methane could be extremely fractionated toward heavy ${ }^{13} \mathrm{C}$ species that values in the $-20 \sim>-10$ per mil, which belong to inorganic methane could be measured.
\end{abstract}

\section{KEY WORD LIST}

Shale gas exploration, quantum cascade laser; laser spectroscopy, Hollow Waveguide; Compound Specific Isotope Analyzer

\section{INTRODUCTION}

Compound specific isotope analysis (CSIA) has many applications in fossil energy exploration ${ }^{[1]}$, food and drug identification, environmental protection and human health care ${ }^{[2]}$. The workhorse instrument for CSIA has been $\mathrm{ga}_{\mathrm{S}}$ chromatograph coupled with Isotope Ratio Mass Spectrometry (GC-IRMS). GC-IRMS has been dedicated $t_{0}$ laboratories and prevented the field application where the fast dynamic measurements, i.e. measurements requiring hours or shorter period, are required. We have been developing such an instrument based on hollow waveguide (HWG) and mid-Infrared quantum cascade (QC) lasers, i.e. Infrared Isotope Ratio (IR2) spectrometer which is based on absorption spectroscopy and coupled with GC, i.e. GC-IR2, to realize Field Deployed CSIA instrument ${ }^{[3-5]}$.

In the past year, our GC-IR2 has been deployed to rig site, and demonstrated the speed, stability and accuracy that enabled the discovery of fast fractionation dynamics, i.e. half time on the scales of one hour to one day, during $\mathrm{sh}_{\mathrm{ale}}$ drilling; whereas such fractionations are hard to observe with GC-IRMS that are tied to laboratory and prevent $\mathrm{su}_{\mathrm{ch}}$ fast real time process to be observed.

\section{EXPERIMENT}

\section{Latest GC-IR2 spectrometer reduce system weight and power consumption}

Recently, noticeable progress has been made in QC lasers in the range of 2,300 $\mathrm{cm}^{-1}$. The devices could $\mathrm{n}_{\mathrm{OW}}$ working in continuous wave (CW) mode at above room temperature up to $70^{\circ} \mathrm{C}^{[6]}$ and with power consumption $1_{\text {ess }}$ than $1 \mathrm{~W}^{[7]}$. These results enable us to replace old $\mathrm{CW}$ QC lasers which need to be cooled down to $-20^{\circ} \mathrm{C}$, and the savings in Peltier cooling power translate into total saving in system temperature control power from $300 \mathrm{~W}$ to only $15 \mathrm{~W}$ ! In fact, we are no longer cooling the spectrometer box and QC lasers, and only small heating power is needed to maintain the spectrometer temperature. With better insulation, we expect the total power to maintain temperature of the spectrometer, including powering and temperature control for the laser, will be reduced to less than $12 \mathrm{~W}$ !.

With the HWG platform, we could pump down the IR2 spectrometer system with a pair of micro diaphragm pum $\mathrm{p}_{\mathrm{p}}$ e.g. Hargraves CTS pump, while maintaining a sample refresh rate higher than $10 \mathrm{~Hz}$. These micro diaphragm pum $\mathrm{ps}$ consume less than $5 \mathrm{~W}$ average power and weigh less than 200 grams.

Quantum Sensing and Nanophotonic Devices XII, edited by Manijeh Razeghi, Eric Tournié, Gail J. Brown, Proc. of SPIE Vol. 9370, 937010 - (C) 2015 SPIE · CCC code: 0277-786X/15/\$18 - doi: 10.1117/12.2080737 
Now, an IR2 spectrometer with total power consumption less than $20 \mathrm{~W}$ and weighs less than 10 pounds is being designed. This opens the possibility to transport the IR system to many places previously impossible, e.g. airborne systems on UAVs.

\section{Improved speed and accuracy for GC-IR2}

With the IR2 spectrometer, we integrate a customized dual oven GC (SRI Instruments, Torrance, CA) to form the GC-IR2 instrument. In the past 2 years, we have developed and improved the performance of the instrument. The instrument is now able to measure $\delta^{13} \mathrm{C}$ with an error $(1 \sigma)$ within \pm 0.06 per mil for a hydrocarbon compound coming out of a GC. After coupling GC, where the sample injection and separation errors are introduced, we could guarantee an error $(1 \sigma)$ within \pm 0.3 per mil for the measurement of methane, $\mathrm{CO} 2$, ethane, propane and butane out of natural gas samples, as demonstrated in Figure 1. The dual oven customized GC speeds up the sampling frequency, so that each natural gas sample could be analyzed in 5 minutes with compound specific values for methane, $\mathrm{CO} 2$, ethane and propane. The improved throughput of the instrument is vital for rig-site sample analysis, where the number samples is usually over 5 samples each hour.

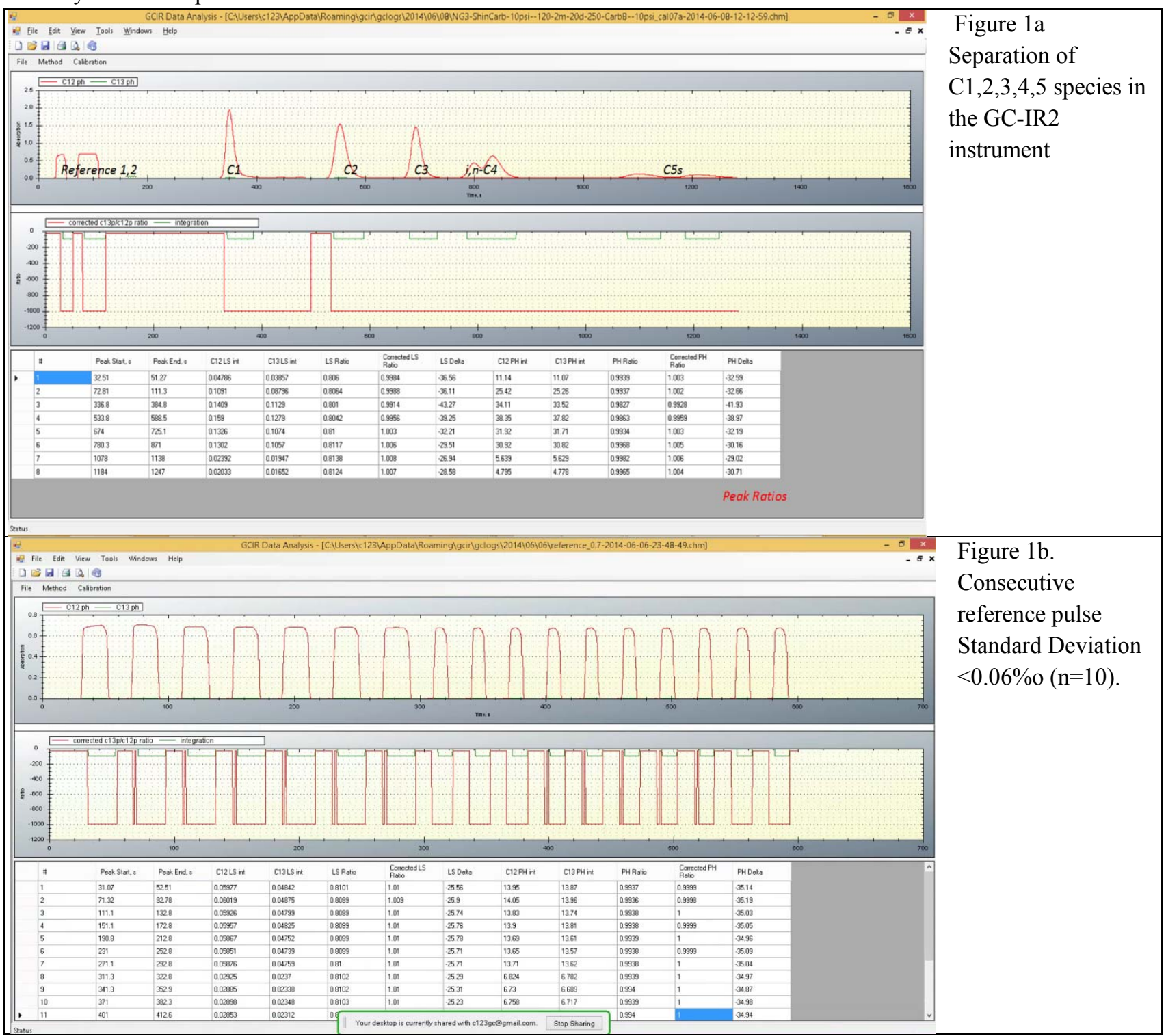




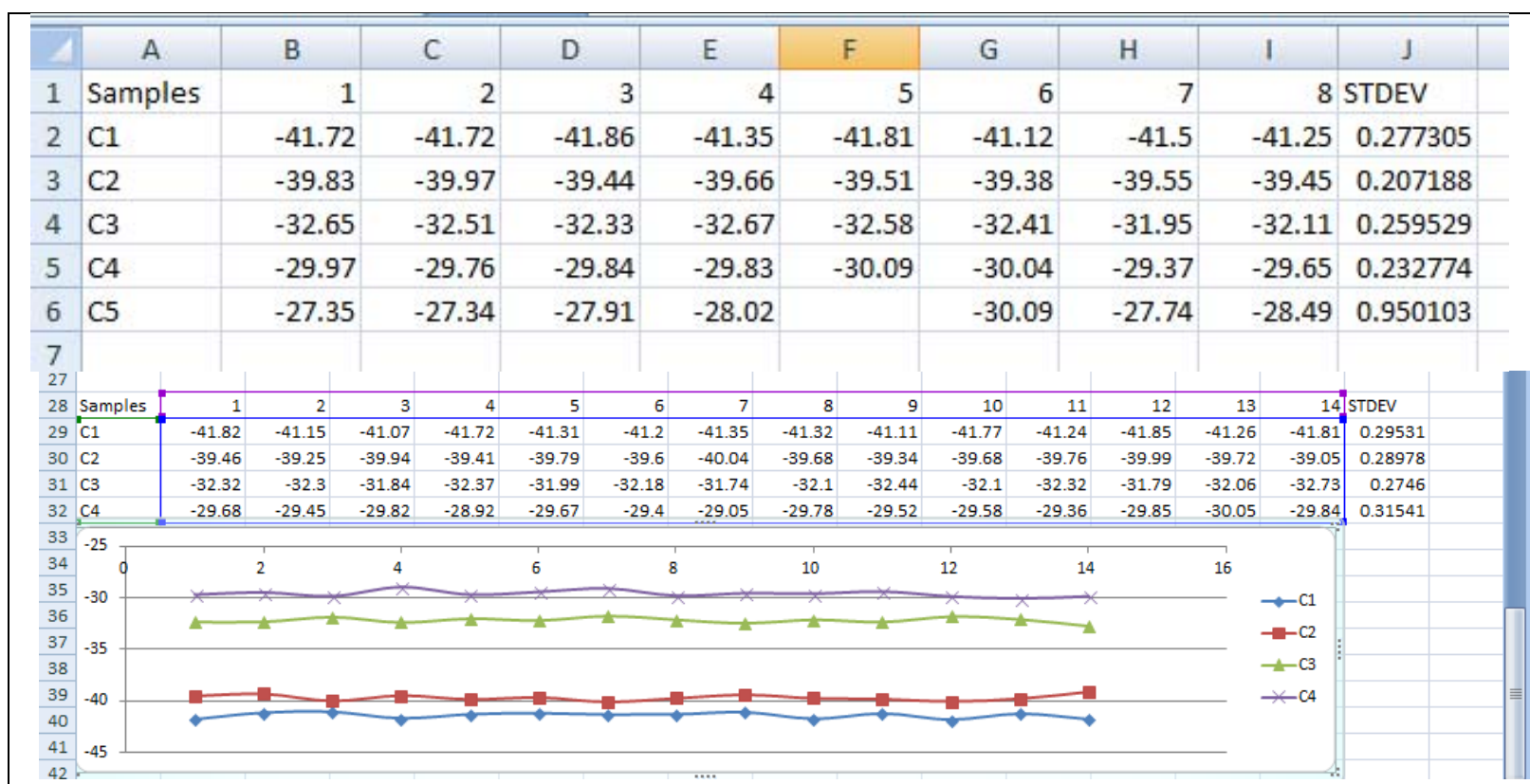

Figure 1c. C1, C2, C3 Standard Deviation $<0.3 \% \mathrm{o}(\mathrm{n}=14)$ Reference pulse standard Deviation $<0.2 \% \mathrm{o}(\mathrm{n}=14)$

\section{RESULTS}

GC-IR2 helped the discovery of fast gas release during shale exploration and sweetspot

In the ongoing shale oil and gas revolution, one key driving the boom or bust of the revolution is the cost of the shale oil and gas versus that of the conventional resources. One of the factors that keep the cost of shale oil and gas from going down further is the ability to find and track the "sweet spots", or the higher yield zones, of the shale reservoirs. These "sweet spots" are characterized by high gas/oil content, and high permeability and porosity, i.e. there are lots of hydrocarbon sources inside and they could come out relatively easily once fracked. Currently, in many of the shale oil and gas fields, even when drilled in the same vertical well, different horizontal/lateral extensions wells toward different directions of the same layer could yield dramatically different yields, survey shows that almost half of the lateral wells drilled could not have enough returns to cover the cost to drill them, while a smaller percentage of laterals drilled ("sweet spot") could have very high returns that well compensate the developer's loss. The key for improving the return rate is to develop log well drilling strategy that could direct the lateral wells stay in the "sweet spots", usually within \pm 10 ' accuracy. The conventional Measure while Drilling (MWD) techniques such as gamma ray, resistivity usually don't work because the shale payzone layers usually extends $10 \mathrm{~s}$ to 100 s of feet thick and nearby layers also have similar resistivity and gamma characterizations. Conventional geochemical well logging tools, such as mud gas logging, also do not work well because the lateral wells stay in the pay zone and baseline is elevated to very high level throughout the drilling process. Techniques are required to identify sweet spots that have both high oil and gas content as well as high porosity and permeability.

It has been proposed that CSIA analysis of methane $\delta^{13} \mathrm{C}$ variations between isotube and isojar, i.e. free mud gas and gas released later from cuttings, could be used to provide such information as porosity and permeability ${ }^{[8]}$. But there are no corroborations of this approach in real shale development. In fact, some drilling results show that the largest variations between isotube and isojar exist, instead of where sweet spot should be, often at the top of the pay zone where the production is not good. 


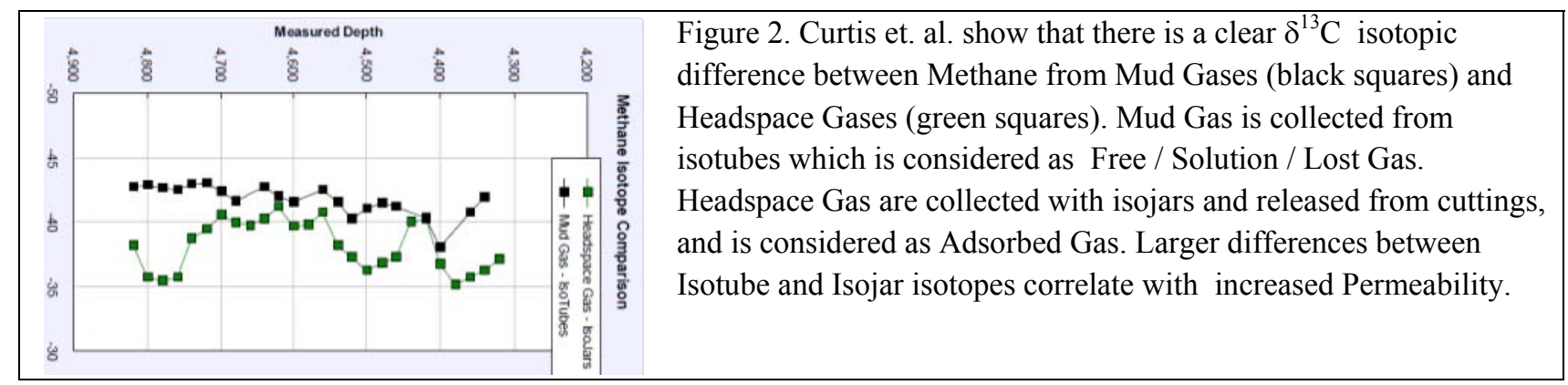

We take the GC-IR2 instrument to shale drilling site in Woodford shale field, OK. We collected isotubes and isojars from different depths above and during the payzone, and conducted CSIA of methane, ethane, propane and $\mathrm{CO} 2$ for both tubes and jars from fresh samples when they are collected only 10 minutes ago till a week later. The tubes' CSIA values remain constant over the measurement campaign, without incidents. We were surprised to see that the jars' CSIA values changes drastically over the week long period. Initially, all jars have CSIA $\delta^{13} \mathrm{C}$ readings for methane in the very positive territory, i.e. - $20 \sim-10 \%$ o range. This range of $\delta^{13} \mathrm{C}$ values belongs to inorganic sources, which seems obviously contradictory to the shale gas that we are drilling. Yet, in as short as several hours, to as long as 6 days, all the ${ }^{13 / 12} \mathrm{C}$ values shifted back in the $<-30 \%$ o range. The lifetime of this shift process, i.e. from hours to as long as 6 days, was not observed before. In this process of hours to as long as 6 days when the shift of ${ }^{13 / 12} \mathrm{C}$ values for methane, ethane and propane, there are also accompanying accumulated release of ethane and propane. We discovered that the shortest period corresponds to the pay zone depth, where the final amount of methane, ethane and propane in the jars are the most abundant. The lifetime for such abundant and fast process period is as short as 30 minutes, indicating that it is imperative to conduct the measurement right at the well site on fresh samples.

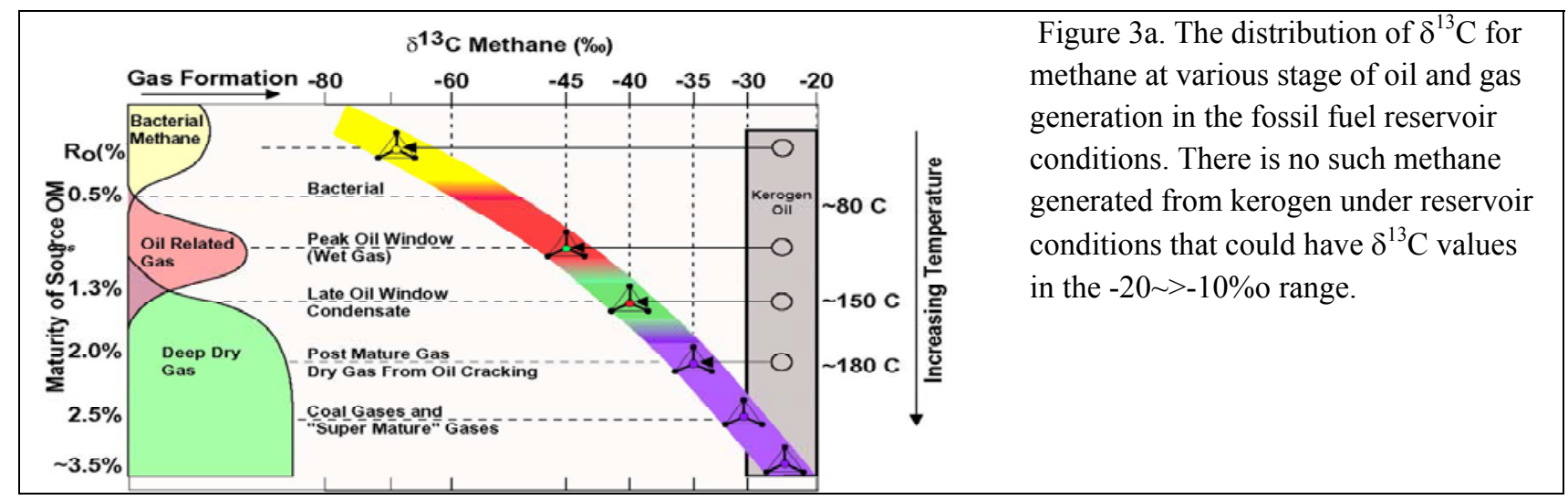




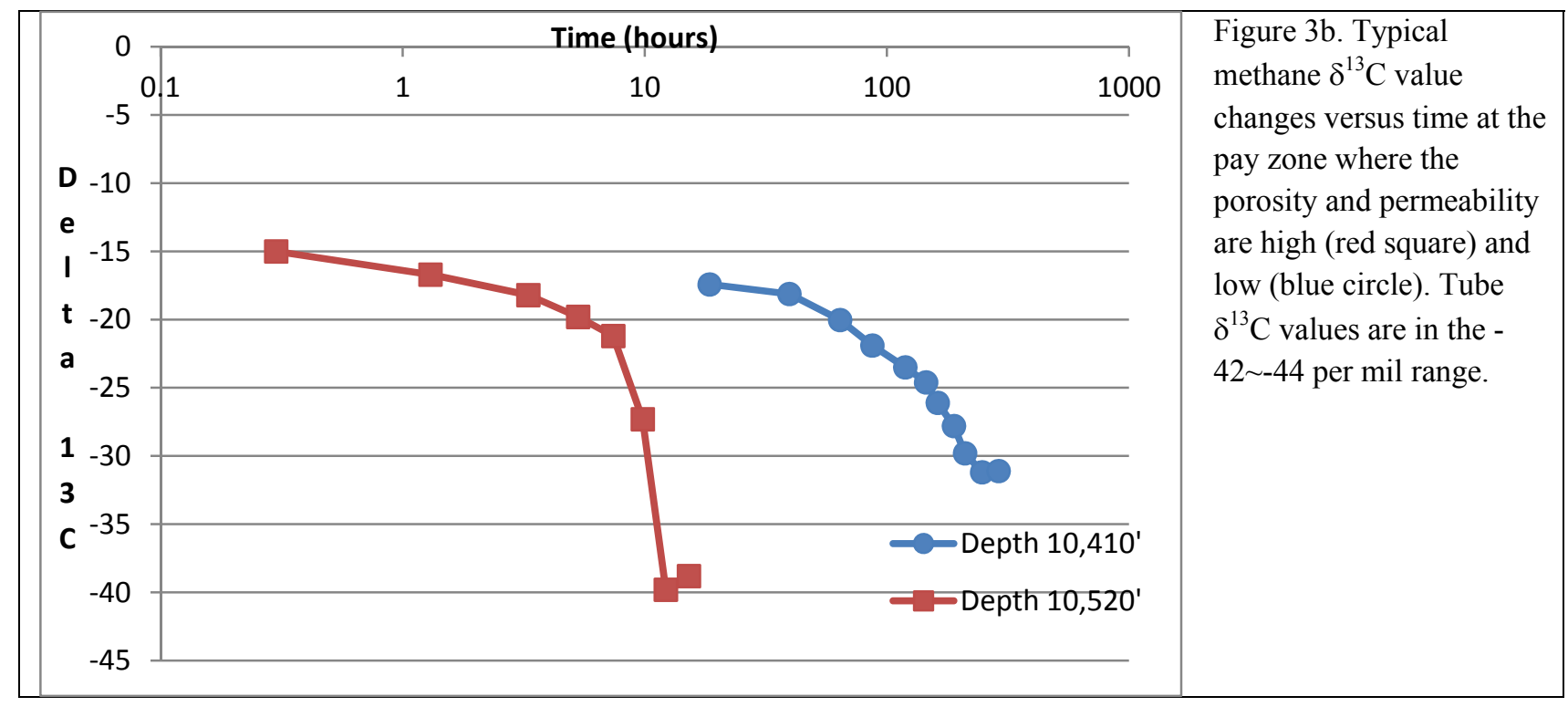

\section{DISCUSSIONS}

\section{Advantages and disadvantages of the GC-IR2 sensor}

GC-IR2 spectrometer is based on strong optical absorption in the Mid-Infrared, therefore, it has advantages and disadvantages over IRMS. The advantages include the easy differentiation of isotopomers with the same mass, e.g. ${ }^{13} \mathrm{CO}_{2}$ versus ${ }^{12} \mathrm{C}^{17} \mathrm{O}^{16} \mathrm{O}$. But, it could not detect molecules without strong IR active absorption features, e.g. $\mathrm{H}_{2}$, or $\mathrm{H}_{2} \mathrm{O}$. The future development of room temperature $\mathrm{THz}$ lasers and detectors in the $200 \mathrm{~cm}^{-1}$ range could enable the detection of $\mathrm{H}_{2} \mathrm{O}$ there with IR2, but not yet practical right now.

The stability of the IR2 spectrometer is inherent due to the underlining optical absorption nature of the instrument. This inherent property lends to the easy observation of fractionation in reference pulses as the conditions of pulse generation are varied.

The precision of the IR2 spectrometer is also getting close to that of IRMS. Although the sample amount needed is still larger than MS, it is already below $10 \mu \mathrm{L}$ (Standard Pressure and Temperature), making it compatible with GC operation. The small volume makes calibration easy to carry out in the field. The small sample volume, i.e. $<10 \mu \mathrm{L}$ (standard temperature and pressure, STP) makes our IR2 spectrometer unique when compared to other well-known optical absorption based instruments with larger sample volumes, e.g. cavity enhanced and multipass cell absorption spectroscopy platforms all have sample volume larger than $1 \mathrm{ml}$ STP.

\section{CONCLUSION}

With the newly developed GC-IR2 instrument, we discovered the fast dynamics of gas release in shale gas/oil drilling. The findings should aid the Log While Drilling (LWD) process to optimize the production of shale lateral drilling. These field test results proved that GC-IR could handle measurements that traditional GC-IRMS could not handle, and its accuracy and speed coupled with field deployment capability should find new applications in energy, environmental, food and health market. 


\section{REFERENCES}

[1] M. Schoell. Genetic-Characterization of Natural Gases. Aapg Bulletin-American Association of Petroleum Geologists 1983, 67, 2225.

[2] A. Sessions. Isotope-ratio detection for gas chromatography. J Sep Sci 2006, 29, 1946.

[3] S. Wu, A. Deev. A Field Deployable Compound Specific Isotope Analyzer based on Quantum Cascade Laser and Hollow Waveguide. SPIE Photonics West Conference Proceeding, Nano and Quantum Sensing 2013, DOI:10.1117/12.2003261.

[4] S. Wu, A. Deev, M. Haught, Y. Tang. Hollow waveguide quantum cascade laser spectrometer as an online microliter sensor for gas chromatography. Journal of Chromatography A 2008, 1188, 327.

[5] Sheng Wu, A. Deev. Latest improvements in field deployable compound specific isotope analyzer based on quantum cascade lasers and hollow waveguide. Proc. SPIE 8993, Quantum Sensing and Nanophotonic Devices XI, 2013, 8993.

[6] X. Feng, C. Caneau, H. P. LeBlanc, N. J. Visovsky, S. Coleman, L. C. Hughes, Z. Chung-en. Room Temperature CW Operation of Mid-IR Distributed Feedback Quantum Cascade Lasers for CO2,N2O, and NO Gas Sensing. Selected Topics in Quantum Electronics, IEEE Journal of 2012, 18, 1605.

[7] B. Hinkov, A. Bismuto, Y. Bonetti, M. Beck, S. Blaser, J. Faist. Singlemode quantum cascade lasers with power dissipation below $1 \mathrm{~W}$. Electronics Letters 2012, 48, 646.

[8] J. E. Zumberge, K. A. Ferworn, J. B. Curtis. Gas character anomalies found in highly productive shale gas wells. Geochimica et Cosmochimica Acta Supplement 2009, 73, pA1539. 